\title{
Avaliação da exposição de passageiros de veículos às partículas ultrafinas em condições de tráfego de um grande centro urbano.
}

\author{
Lilian L. N. Guarieiro ${ }^{1,5^{*}}$, Egídio T. A. Guerreiro ${ }^{1,5}$, Paulo A. Pinho ${ }^{1}$, Rodrigo P. Vieira ${ }^{1}$, \\ Jailson B. de Andrade ${ }^{2,4,5}$, Antonio H. Miguel ${ }^{6}$
}

${ }^{1}$ SENAI CIMATEC , 41650-010, Salvador-BA, Brasil;

${ }^{2}$ Universidade Federal da Bahia, Instituto de Química, 40170290 Salvador, BA, Brasil;

${ }^{3}$ Universidade Federal da Bahia, Escola Politécnica, 40210-730 Salvador, BA, Brasil;

${ }^{4}$ Centro Interdisciplinar de Energia e Ambiente (CIEnAm), 40110-040 Salvador, BA, Brasil;

${ }^{5}$ INCT de Energia e Ambiente, UFBA, 40170-290 Salvador, BA, Brasil

${ }^{6}$ Particle Center and Supersite, Institute of the Environment, University of California, Los

Angeles, 90095, CA, USA (atualmente na Califórnia Environmental Protection Agency).

\author{
E-mails: *lilianguarieiro@gmail.com
}

\section{RESUMO}

Existem evidências de impactos adversos à saúde, decorrentes da exposição humana relacionada à poluição de partículas ultrafinas (da $<100 \mathrm{~nm}$ ), oriundas do tráfego urbano. À medida que mais passageiros estão gastando uma parcela significativa de sua rotina diária dentro de veículos, é cada vez mais relevante o estudo dos níveis de exposição a poluentes nocivos no interior do veículo. Assim, o objetivo deste trabalho foi avaliar a exposição de passageiros a partículas ultrafinas $(\mathrm{da}=100 \mathrm{~nm})$ no interior de veículos nas condições de tráfego da cidade de Salvador-BA. Um contador de partículas modelo 3910 TSI foi utilizado para determinar a concentração de partículas de $100 \mathrm{~nm}$ no interior de um veículo. A exposição de passageiros a partículas ultrafinas foi avaliada em 5 regiões de Salvador, com diferentes condições de tráfegos. O sitio com baixo fluxo de ar e alto fluxo de veículos pesados foi o que apresentou maior faixa concentração de partículas $(100 \mathrm{~nm})$ no interior do veículo $\left(0,6 \mathrm{E}+06\right.$ a $\left.1,4 \mathrm{E}+07 \mathrm{\#} / \mathrm{cm}^{3}\right)$ e o sito com alto fluxo de ar e baixo fluxo de veículos apresentou menor a faixa de concentração $\left(0,3 E+05\right.$ a $\left.0,3 E+06 \# / \mathrm{cm}^{3}\right)$. Além disso, quanto maior o nível de radiação solar, maior poderá ser o nível de exposição dos passageiros às partículas.

\section{INTRODUÇÃO}

O material particulado (MP) oriundo da combustão de motores de combustão interna é constituído de grandes aglomerados carbonáceos sólidos, compostos de enxofre e compostos orgânicos voláteis. O MP é o principal agente na causa e no agravamento de doenças crônicas do aparelho respiratório dos seres humanos, tal toxicidade depende da composição dessas partículas e principalmente do seu diâmetro aerodinâmico. As partículas consideradas ultrafinas, ou seja, quando o seu diâmetro é inferior ou igual a $100 \mathrm{~nm}$, as quais são capazes de ultrapassar as barreias do aparelho respiratório dos seres humanos e alcançar a corrente sanguínea, são consideradas as mais nocivas dentre todas as outras faixas de tamanho de partículas emitidas por motores de combustão interna [1]. 
Alguns estudos têm sugerido que o número de partículas (partículas ultrafinas, faixa nanométrica) está mais relacionado com estes efeitos, do que a massa do material particulado [2,3]. Devido tais efeitos, alguns países priorizam um controle mais rigoroso das partículas emitidas na exaustão veicular, avaliando o número de partículas emitidas na queima. $\mathrm{Na}$ União Européia alguns regulamentos que normatizam o controle do número de partícula já estão sendo introduzidos para veículos pesados movidos a diesel, através da Comissão Européia [4].

O tamanho das partículas é também um fator importante a ser destacado, pois este pode estar diretamente relacionado ao seu potencial em causar problemas à saúde. Pode-se dizer que quanto menor o tamanho das partículas, maiores são os efeitos que estas podem causar na saúde. Assim, o tamanho que as partículas são encontradas na atmosfera vai depender de suas fontes, processos de formação, e a superfície onde estas partículas são formadas [5]. Existem três grupos distintos de classificação das partículas: grossa, fina e ultrafina ou nanopartículas.

As partículas atmosféricas são caracterizadas de acordo com seu diâmetro aerodinâmico (dp), $\mathrm{PM}_{0.1}$ ultrafinas $(\mathrm{dp}<100 \mathrm{~nm}), \mathrm{PM}_{2.5}$ finas $(\mathrm{dp}<2,5 \mu \mathrm{m})$, grossas $\mathrm{PM}_{10-2.5}(\mathrm{dp} \geq 2,5 \mu \mathrm{m} \mathrm{e}<$ $10 \mu \mathrm{m})$ ou $\mathrm{PM}_{10}(\mathrm{dp}<10 \mu \mathrm{m})$ podem ser oriundas de fontes naturais e antropogênicas [6]. Os padrões atuais de qualidade do ar são baseados nas concentrações de massa total de material partículado. Assim, as concentrações de menor massa (as nanopartículas, que realmente dominam a concentrações de partículas totais em áreas urbanas) tornam-se insignificantes [7].

Atualmente, a Organização Mundial de Saúde menciona que ainda não existem evidências suficientes que estabeleçam os efeitos nocivos das nanopartículas à saúde [8]. No entanto, o número de evidências epidemiológicas é insuficiente para concluir sobre níveis toleráveis de exposição destas partículas para a saúde [9]. Recentemente, no Brasil, especulações estão sendo feitas a respeito, para que a norma de certificação de veículos inclua o teste do número de partículas emitidas por veículos leves. Essa preocupação se baseia no efeito que as nanopartículas podem trazer à nossa saúde.

Neste contexto, pode-se citar como fontes principais de nanopartículas na atmosfera as emissões de transportes, tais como automóveis, aviões, navios e outros. Este dado se confirma, através de pesquisa que são realizadas para este tamanho de partícula durante medições em túneis rodoviários, rodovias e vias urbanas [10-15].

A distribuição de tamanho de partículas têm sido objeto de estudos em várias cidades da Europa e Estados Unidos, por exemplo, Birmingham [16], Atlanta [17], Helsinki [18], Leipzig [19] e Pittsburgh [7]. As partículas oriundas dos processos de combustão veicular, mais especificamente do óleo diesel, são constituídas de grandes aglomerados carbonáceos sólidos, compostos de enxofre e compostos orgânicos voláteis (COV) [10]. As partículas emitidas na combustão de motores veiculares podem estar distribuídas em diferentes faixas de tamanho e número de partículas. As partículas distribuídas na faixa de tamanho que abrange as nanopartículas, possuem um potencial toxicológico maior que partículas maiores [20].

Em termos de exposição de passageiros à poluição veicular, estudos apontam para o fato que os efeitos de barreiras (janela e ventilação) na cabine do veículo são os principais determinantes das concentrações de exposição do material particulado no veículo [21,22]. Assim, estudos que quantifiquem o nível de exposição dos passageiros podem estabelecer os efeitos de trânsito nas partículas ultrafinas e as relações não-lineares entre tráfego e 
concentrações variáveis destas partículas, trazendo implicações importantes para modelagem e potenciais estratégias de mitigação da exposição ao material particulado oriundo da exaustão veicular.

Nesse sentido, o presente trabalho teve como objetivo avaliar a exposição de passageiros a partículas ultrafinas $(\mathrm{dp}=100 \mathrm{~nm})$ no interior de um veículo em condições típicas de tráfego da Região Metropolitana de Salvador (RMS).

\section{MATERIAS E MÉTODOS}

Um contador de partículas (modelo 3910 TSI com faixa de medição de 10-420 nm, sendo capaz de medir concentrações de até 1.000 .000 particulas $/ \mathrm{cm}^{3}$ ) foi utilizado para determinar a concentração de partículas de $100 \mathrm{~nm}$ no interior de um veículo Toyota Hilux 3.0 (diesel, 4x4, ano 2010). O contador de partículas foi posicionado no centro do banco traseiro e um termohigrometro digital (Minipa MT240) foi utilizado para medir a temperatura e umidade de cada coleta.

A exposição de passageiros a partículas ultrafinas foi avaliada em 5 regiões de RMS com diferentes condições de tráfegos: (sitio A) percurso com alto fluxo de veículos e sem corredores de edifícios; (sitio B) percurso com alto fluxo de veículos, sem corredores de edifícios e alto impacto de spray marino, avenida oceânica; (sitio C) percurso com baixo fluxo de veículos e sem corredores de veículos; (sitio D) percurso com alto fluxo de veículos e com corredores de edifícios; e (sitio E) percurso com alto fluxo de veículos e ambiente fechado (Túnel) (Tabela 1).

Tabela 1 - Resumo dos percursos de amostragens

\begin{tabular}{cll}
\hline Sitio & \multicolumn{1}{c}{ Região } & \multicolumn{1}{c}{ Característica } \\
\hline A & Av. Paralela & Ambiente aberto com alto fluxo de veículos. \\
B & Av. Oceânica & Ambiente com alto fluxo de ar. \\
C & Centro Adm. da Bahia & Ambiente com alto fluxo de ar. \\
D & Av. Tanc. Neves & Ambiente fechado com baixo fluxo de ar. \\
E & Túnel & Ambiente com alta circulação de veículos leves e pesados, e pouquíssimo \\
\hline
\end{tabular}

As amostras foram coletadas em três diferentes horários do dia $(9: 00 \mathrm{~h}, 13: 00 \mathrm{~h}$ e 17:00 h), para avaliar a influência da radiação solar e fluxo de veículos em horários de congestionamentos. O planejamento de coleta foi estabelecido para ser executado em duas condições diferentes: Janelas totalmente abertas; Janelas fechadas sem ar condicionado. Cada percurso durou em média 3 minutos. Os dados das condições dos sítios estão descritos na Tabela 2.

Tabela 2. Condição de trafego dos sítios estudados.

\begin{tabular}{cccccccc}
\hline Sitio & $\begin{array}{c}\text { Horário } \\
\text { inicial }\end{array}$ & $\begin{array}{c}\text { Horário } \\
\text { final }\end{array}$ & Transito & $\begin{array}{c}\text { T oC } \\
\text { inic/final }\end{array}$ & $\begin{array}{c}\text { UR } \\
\text { inic/final }\end{array}$ & $\begin{array}{c}\text { Veículos } \\
\text { leves }\end{array}$ & $\begin{array}{c}\text { Veículos } \\
\text { pesados }\end{array}$ \\
\hline \multirow{3}{*}{$\mathrm{A}$} & $09: 40$ & $10: 36$ & Razoável & $29 / 31$ & $60 / 57$ & 78 & 6 \\
& $13: 03$ & $13: 50$ & Intenso & $28 / 30$ & $50 / 52$ & 80 & 12 \\
& $17: 02$ & $17: 43$ & Intenso & $27 / 29$ & $50 / 52$ & 84 & 15 \\
\hline \multirow{2}{*}{ B } & $09: 02$ & $09: 29$ & Livre & $31 / 32$ & $68 / 68$ & 42 & 8 \\
& $13: 00$ & $13: 25$ & Livre & $31 / 32$ & $71 / 69$ & 64 & 12 \\
& $17: 05$ & $17: 34$ & Livre & $30 / 31$ & $75 / 74$ & 72 & 16 \\
\hline \multirow{2}{*}{$\mathrm{C}$} & $09: 08$ & $10: 00$ & Livre & $30 / 29$ & $49 / 49$ & 36 & 1 \\
& $12: 53$ & $13: 34$ & Livre & $29 / 30$ & $53 / 52$ & 32 & 3
\end{tabular}




\begin{tabular}{cccccccc} 
& $17: 02$ & $17: 43$ & Livre & $27 / 28$ & $49 / 47$ & 30 & 2 \\
\hline \multirow{3}{*}{ E } & $09: 16$ & $09: 41$ & Razoável & $30 / 31$ & $46 / 42$ & 102 & 21 \\
& $13: 10$ & $13: 55$ & Razoável & $31 / 32$ & $50 / 49$ & 122 & 16 \\
& $16: 54$ & $17: 11$ & Razoável & $29 / 30$ & $67 / 67$ & 144 & 19 \\
\hline \multirow{3}{*}{ D } & $09: 20$ & $09: 45$ & Razoável & $29 / 28$ & $62 / 63$ & 84 & 12 \\
& $13: 14$ & $14: 02$ & Razoável & $30 / 31$ & $55 / 58$ & 69 & 14 \\
\hline
\end{tabular}

\section{RESULTADOS E DISCURSÕES}

As Figuras 1 e 2 apresentam os resultados obtidos no presente estudo para exposição de passageiros com as janelas abertas e janelas fechadas do veículo. Os pontos ilustrados nos gráficos apresentam a concentração média de número de partículas de $100 \mathrm{~nm}$ obtida em cada sítio e as barras de erros apresentam os valores máximos e mínimos obtidos.

Para as coletas com as janelas abertas, onde é possível avaliar o impacto máximo que os passageiros podem sofrer com a exposição à poluição oriunda dos sítios estudados, pode-se notar que os sítios que apresentaram maior impacto foram os sítios E e D (alto fluxo de veículos e baixo fluxo de ar. Tais resultados já eram esperados, uma vez que a característica destes sítios (alto fluxo de veículos pesados e baixo fluxo de ar), possuem uma contribuição maior na emissão de partículas ultrafinas.

$\mathrm{O}$ sítio $\mathrm{E}$ foi o local onde o passageiro pode sofrer o maior impacto das partículas nanométricas $(100 \mathrm{~nm})$. Foi observado maior faixa de concentração de partículas $(100 \mathrm{~nm})$ no interior do veículo $\left(0,6 \mathrm{E}+06\right.$ a $\left.1,4 \mathrm{E}+07 \# / \mathrm{cm}^{3}\right)$, isso é devido às características físicas do percurso que não proporcionam a ventilação, impedindo a dissipação dos poluentes emitidos pelos veículos. Assim, características deste local de amostragem (Túnel) influenciam diretamente na dispersão das partículas emitidas na queima de combustíveis veiculares, uma vez que este é um local completamente fechado. Já o sítio D, apesar de não ser um local completamente fechado, possui corredores de edifícios em toda sua extensão, o que também dificulta a dispersão das partículas emitidas do escapamento dos veículos. Dessa forma, este sitio também apresentou concentrações elevadas de número de partículas (100 nm), quando comparado com os demais locais.

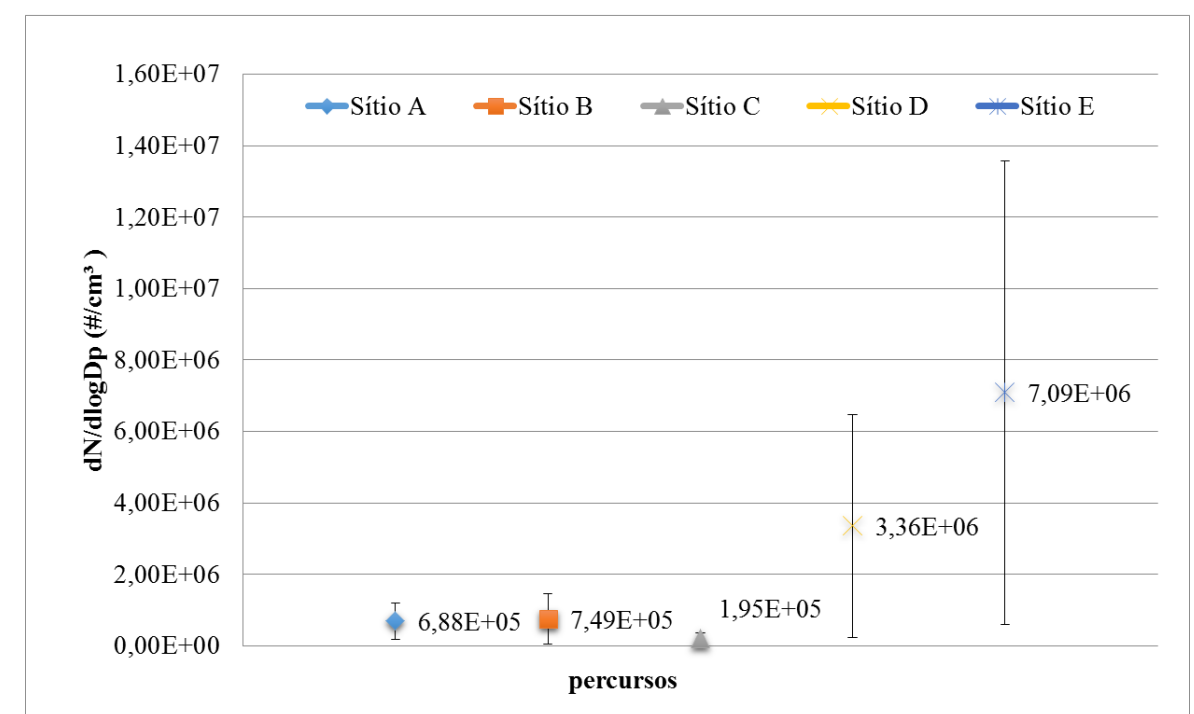

Figura 1. Concentração de partículas $(100 \mathrm{~nm})$ na cabine de veículos com as janelas totalmente abertas. 
Nos sítios A e B foram observadas concentrações menores de número de partículas quando comparados com os sitíos $\mathrm{E}$ e $\mathrm{D}$ e o sítio $\mathrm{C}$ foi o ambiente que apresentou menor impacto aos passageiros de veículos automotivos. Tais resultados estão de acordo com as características de cada local, tanto em termos de fluxos de veículos pesados, como em termos de características estruturais/ambientais locais.

A Figura 2 apresenta as concentrações das partículas $(100 \mathrm{~nm})$ obtidas nas coletas com as janelas totalmente fechadas. $O$ intuito desta amostragem foi verificar qual a incluencia da presença dos tripulantes do veículo (3) e as possíveis interferências e impactos que as partículas $(100 \mathrm{~nm})$ já existentes no ar interior do veículo poderiam trazer a amostragem e a exposição aos passageiros, caso estes fechassem as janelas próximo a ambientes de alto fluxo de veículos.

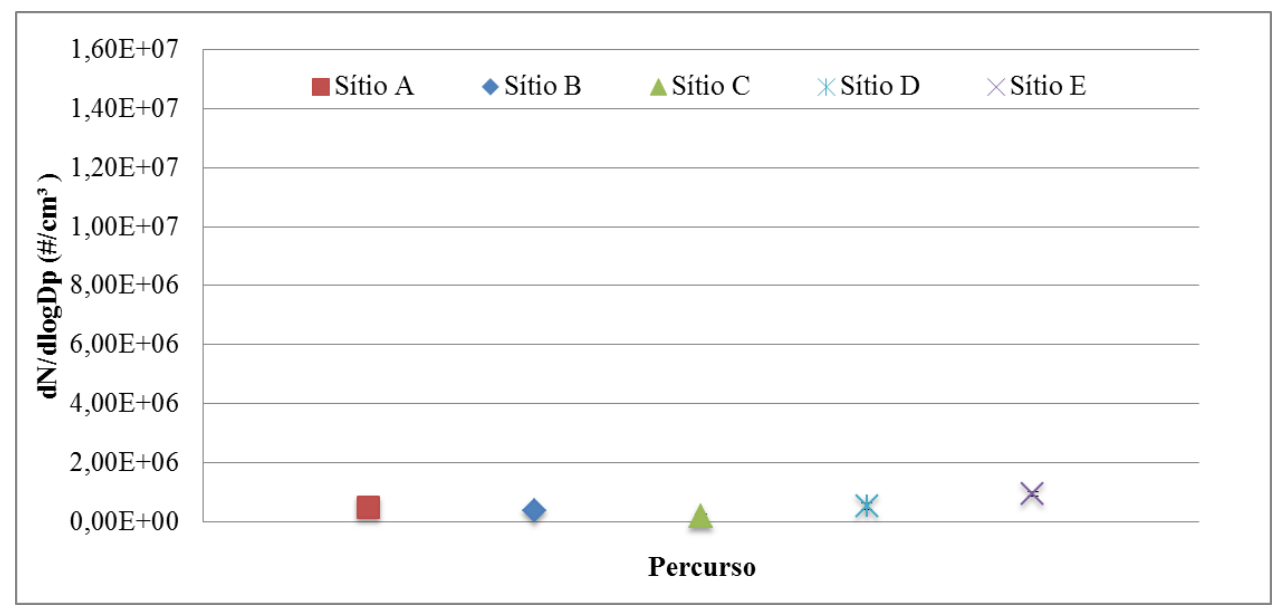

Figura 2. Cncentração de partículas $(100 \mathrm{~nm})$ na cabine de veículos com as janelas totalmente fechadas, sem ventilação.

Pode-se notar que os dados apresentados na Figura 2 direcionam os tripulantes de veículos a fecharem suas janelas caso estes estejam passando por regiões de alto fluxo de veículos. A Figura 3 apresenta um estudo comparativo dos dois tipos de coleta (janelas fechadas e abertas), onde é possível verificar a importância em se trafegar em regiões de alto fluxo de veículos e pouca ventilação com as janelas totalmente fechadas. 


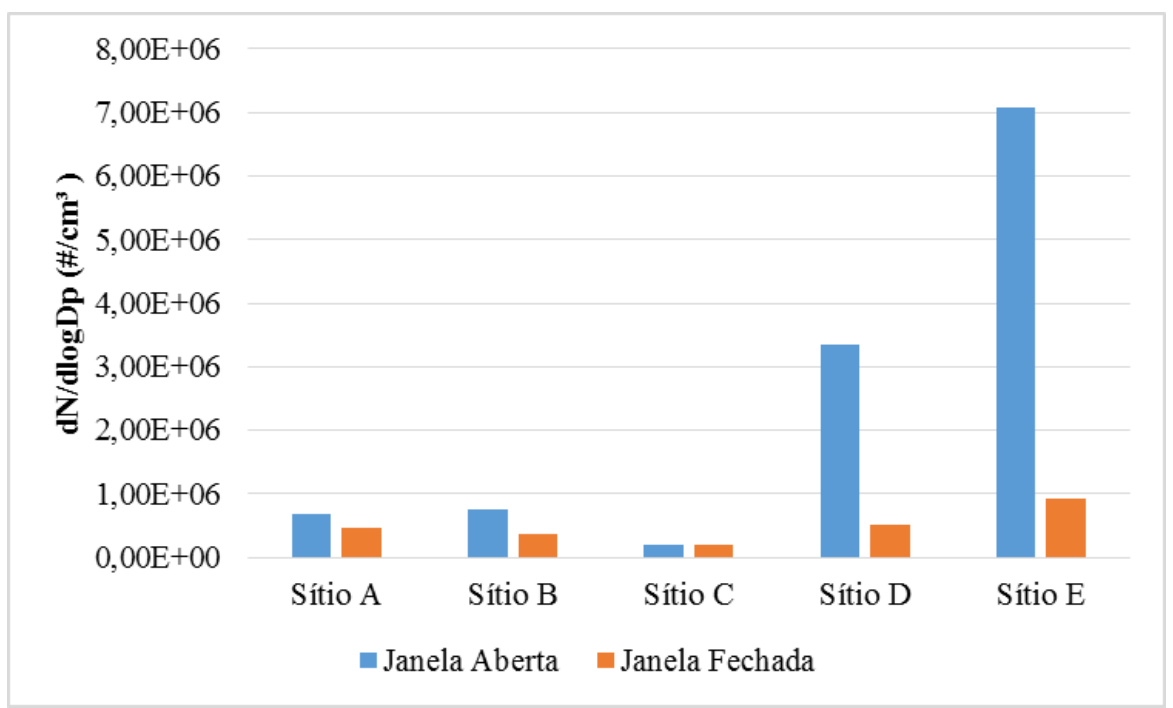

Figura 3. Concentração de partículas obtidas em condições diferentes de amostragem (janelas abertas e janelas fechadas)

De uma forma geral, pode-se dizer que os passageiros de veículos automotores são expostos a altas concentrações de partículas ultrafinas $(100 \mathrm{~nm}$, nominal) em locais com alto fluxo de veículos e baixo fluxo de ar. Os dados obtidos em diferentes horários do dia, com diferentes níveis de radiação mostrou influenciar a concentração número de partículas. Quanto maior o nível de radiação solar, maior poderá ser o nível de exposição dos passageiros às partículas.

\section{CONCLUSÃO}

Os condutores de veículos devem sempre dirigir com as janelas fechadas em locais de alta circulação de veículos e baixo fluxo de ar para reduzir a exposição ao material particulado e gasoso oriundo do escapamento dos veículos, e da ressuspensão da poeira da rua.

\section{AGRADECIMENTOS}

Os autores agradecem ao Instituto Nacional de Ciência e Tecnologia de Energia e Ambiente (INCT EA); Projeto Bahia de Todos os Santos (Portal Kirimurê), ao Conselho Nacional de Desenvolvimento Científico e Tecnológico (CNPq) e a Fundação de Amparo à Pesquisa do Estado de São Paulo (FAPEB).

\section{REFERÊNCIAS}

[1] GUARIEIRO, L.L.N.; VASCONCELlOS, P.C.; Solci, M.C. Poluentes Atmosféricos Provenientes da Queima de Combustíveis Fósseis e Biocombustíveis: Uma Breve Revisão. Revista Virtual de Química. v. 3(5), p. 434-445, 2011

[2] POPE E DOCKERY. Journal of the Air \& waste Management association. 2006, 56, 709.

[3] IMTENAN, S.; MASJUKI, H.H.; VARMAN, M.; KALAM, M.A.; ARBAB, M.I.; SAJJAD, H.; ASHRAFUR RAHMAN, S.M. Impact of oxygenated additives to palm and 
jatropha biodiesel blends in the context of performance and emissions characteristics of a light-duty diesel engine, Energy Conversion and Management, v. 83, p. 149-158, 2014.

[4] JUDA-REZLER, K.; REIZER, M.; OUDINET, J.P. Determination and analysis of PM10 source apportionment during episodes of air pollution in Central Eastern European urban areas: The case of wintertime 2006, Atmospheric Environment, v. 45, p. 6557-6566, 2011.

[5] Morawska et al., Atmos. Environ. 2008. 42, 8113.

[6] Finlayson-Pitts e Pitts 2000. Chemistry of the Upper and Lower Atmosphere - Theory, Experiments, and Applications.

[7] Stanier et al., Atmospheric Environment. 2004. 38, 3275.

[8] WHO, World Health Organization, 2005. Guidelines for Air Quality.World Health Organization, Genova.

[9] Tartakovsky et al., Atmospheric Environment. 2013. 64, 320.

[10] Martins et al., Environ Monit Assess. 2011, in press.

[11] Da Rocha et al., Journal of the Brazilian Chemical Society. 2009. 20, 680.

[12] Vogt et al., Journal of Aerosol Science. 2003. 34, 319.

[13] Sturm et al., Atmospheric Environment. 2003. 37, 5273.

[14] Pirjola et al., Atmospheric Environment. 2004. 38, 3625.

[15] Westerdahl et al., Atmospheric Environment. 2005. 39, 3597.

[16] Harrison et al., Atmospheric Environment. 1999. 33, 309.

[17] Woo et al., Aerosol Science and Technology. 2001. 34, 75.

[18] Hussein et al., Atmospheric Chemistry and Physics. 2004. 4, 391.

[19] Wehner e Wiedensohler, Atmospheric Chemistry and Physics. 2003. 3, 867.

[20] Johansson et al., Atmospheric Environment. 2009. 43, 4681

[21] Bigazzi and Figliozzi, Atmospheric Environment 2012. 60, 495.

[22] Joodatnia et al., Atmospheric Environment. 2013. 71, 364. 